\title{
THE USE OF PROJECTIVE SPACE IN THE STUDY OF POLYNOMIAL VECTOR FIELDS AT INFINITY*
}

\author{
BY ENRIQUE A. GONZÁLEZ-VELASCO (University of Lowell, Lowell, Mass.)
}

1. Introduction. We shall consider polynomial vector fields in Euclidean space $R^{k}$ and show that, when the degree of the polynomials is odd, it is possible to obtain an analytic vector field induced on the projective $k$-space $P^{k}$ by the original one. Thus, the study of this field on the noncompact manifold $R^{k}$ is reduced to that of a field on the compact manifold $P^{k}$.

As an example, we consider the field $X(x, y)=\left(y, \epsilon\left(1-x^{2}\right) y-x\right), \epsilon>0$, corresponding to van der Pol's equation. By studying the region of attraction of one of the nodes at infinity, some plane regions can be found through which the unique limit cycle of this field cannot pass.

2. The two-dimensional case. The study of plane polynomial vector fields in a neighborhood of infinity was initiated by Poincaré in his celebrated Mémoire of 1881 [1] in the following way. The plane is imbedded in 3-space in such a way that it is tangent to the unit sphere $S^{2}$ at the north pole. Then, by gnomonic projection of the trajectories of the original field onto $S^{2}$, an induced vector field is obtained on $S^{2}-S^{1}$, where $S^{1}$ is the equator of $S^{2}$.

In a previous paper [2] we have presented this method of Poincaré and its extension to the $k$-dimensional case in modern terminology, and have shown that the induced field obtained above can be extended analytically to the whole of $S^{2}$ after multiplication by a suitable factor which depends only on the degree of the polynomials in the original field. The extension is such that $S^{1}$, which represents the points at infinity, is an invariant set.

Lefschetz has suggested [3] that Poincaré's method amounts to transforming the original differential equation in the plane into another in the projective plane. What Lefschetz actually constructs is a line element field, but if a vector field is desired some difficulties arise when the degree of the polynomials is even. For example, the trajectories of the vector field $X(x, y)=\left(x^{2}, 0\right)$ which are not critical points are parallel horizontal lines, the direction of motion being towards the right; that is, these trajectories tend to the point at infinity in the direction of $y=0$. Therefore, this point should be a node, but the question of whether this node is contracting or expanding is not easy to answer. Everything happens as if the trajectories tend to the "right half" of this point at infinity and leave from the "left half".

The purpose of this paper is to carry out a detailed analysis of the use of the projective plane. We shall show that, if the degree of the polynomials is odd, then it is possible to obtain an induced field on the projective plane which is analytic. Then we extend this result to the $k$-dimensional case. Finally, as an example, we consider van der Pol's equation and determine some plane regions through which its unique limit cycle cannot pass.

\footnotetext{
* Received March 19, 1979.
} 
The starting point in our analysis will be to induce a vector field on the projective plane $P^{2}$ except on the line at infinity. First, we identify $P^{2}$ with the set of all straight lines through the origin in $R^{3}$. If $y=\left(y_{1}, y_{2}, y_{3}\right) \in R^{3}-\{0\}$, then the line through $y$ will be denoted by $l(y)$. Then, $P^{2}=\{l(y)\}$.

We now consider the following differentiable structure on $P^{2}$. The neighborhoods are given by $U_{i}=\left\{l(y) \in P^{2}|\forall y \in l(y), j \neq i,| y_{j} / y_{i} \mid<\infty\right\}$, and the corresponding maps $\varphi_{i} 1: 1 U_{i} \rightarrow R^{2}$ are defined by

$$
\varphi_{i}(l(y))=\left(y_{j}, y_{k}\right) / y_{i}, \quad i, j, k=1,2,3 ; \quad j<k .
$$

The value of $\varphi_{i}(l(y)) \in R^{2}$ will be denoted by $z=(s, t)$ for any $i$, so that $z$ represents different things depending on the case under consideration.

Next, define the following function $f$ from our original plane into $P^{2}$. If $x=\left(x_{1}, x_{2}\right)$ is a point in the plane, then

$$
f(x)=\left\{l(y) \in P^{2} \mid y_{1} / y_{3}=x_{1}, y_{2} / y_{3}=x_{2}\right\} .
$$

We need the derivative of $f$ in order to obtain the induced field. If $f(x) \in U_{1}$, then (1) and (2) imply that

$$
z=\varphi_{1}(l(y))=\left(y_{2}, y_{3}\right) / y_{1}=\left(x_{2}, 1\right) / x_{1} .
$$

The derivative of $f$ is represented by the matrix $\left(D f_{x}\right)_{i j}=\partial z_{i} / \partial x_{j}$, and, consequently,

$$
D f_{x}=\left[\begin{array}{cc}
-x_{2} / x_{1}{ }^{2} & 1 / x_{1} \\
-1 / x_{1}{ }^{2} & 0
\end{array}\right]
$$

Then, if $X=(P, Q)$ is the original field in the plane, we have

$$
\begin{aligned}
D f_{x}(X(x)) & =\left(1 / x_{1}\right)^{2}\left[x_{1} Q(x)-x_{2} P(x),-P(x)\right] \\
& =\left(\frac{y_{3}}{y_{1}}\right)^{2}\left[\frac{y_{1}}{y_{3}} Q\left(\frac{y_{1}}{y_{3}}, \frac{y_{2}}{y_{3}}\right)-\frac{y_{2}}{y_{3}} P\left(\frac{y_{1}}{y_{3}}, \frac{y_{2}}{y_{3}}\right),-P\left(\frac{y_{1}}{y_{3}}, \frac{y_{2}}{y_{3}}\right)\right] .
\end{aligned}
$$

This field is analytic at all points of $U_{1}$ except (when $n>1$ ) at those points for which $y_{3}=$ 0 , that is, those which correspond to points at infinity.

Next we shall look for a correcting factor such that multiplication of (5) by this factor results in a field without the singularities mentioned above. This factor will depend on the degree $n$ of the original vector field $X$, and its application should not alter the trajectories and critical points of the induced field. Moreover, in terms of the coordinates the $P^{2}$, our factor $F\left(y_{1}, y_{2}, y_{3}\right)$ must be such that $F\left(\lambda y_{1}, \lambda y_{2}, \lambda y_{3}\right)=F\left(y_{1}, y_{2}, y_{3}\right)$ for all real $\lambda$. Therefore, we can choose $F\left(y_{1}, y_{2}, y_{3}\right)=\left[y_{3}{ }^{2} /\left(y_{1}{ }^{2}+y_{2}{ }^{2}+y_{3}{ }^{2}\right)\right]^{(n-1) / 2}$. With $z=(s, t)$ as given by (3) and putting $\Delta(s, t)=\left(1+s^{2}+t^{2}\right)^{1 / 2}$, this factor can be rewritten as $\left[t^{2} /\left(1+s^{2}\right.\right.$ $\left.\left.+t^{2}\right)\right]^{(n-1) / 2}=\left(t^{2}\right)^{(n-1) / 2} / \Delta(s, t)^{n-1}$.

Suppose first that $n$ is odd. Then $\left(t^{2}\right)^{(n-1) / 2}=t^{n-1}$, and multiplication of (5) by the proposed factor leads to the final expression for the induced field on $U_{1} \subset P^{2}$ :

$$
\frac{t^{n}}{\Delta(s, t)^{n-1}}\left[Q\left(\frac{1}{t}, \frac{s}{t}\right)-s P\left(\frac{1}{t}, \frac{s}{t}\right),-t P\left(\frac{1}{t}, \frac{s}{t}\right)\right] .
$$

Straightforward modifications give the following expressions for the induced field on $U_{2}$ 
and $U_{3}$ respectively:

$$
\begin{gathered}
\frac{t^{n}}{\Delta(s, t)^{n-1}}\left[P\left(\frac{s}{t}, \frac{1}{t}\right)-s Q\left(\frac{s}{t}, \frac{1}{t}\right),-t Q\left(\frac{s}{t}, \frac{1}{t}\right)\right], \\
\frac{1}{\Delta(s, t)^{n-1}}(P(s, t), Q(s, t)) .
\end{gathered}
$$

It is clear that this induced field is analytic and that the line at infinity, represented by $t=$ 0 in (6) and (7), is an invariant set.

These expressions for the field induced on $P^{2}$ are identical to the ones obtained when using $S^{2}$. The analysis of critical points and closed orbits at infinity (the latter are only possible when $n$ is odd) also applies here as is.

However, if $n$ is even the factor $\left(t^{2}\right)^{(n-1) / 2}=|t|^{n-1}$ is not differentiable at $t=0$, the line at infinity, so that an extension of (5) by means of this factor is impossible. For example, if we take (6) with $t^{n}$ replaced by $t|t|^{n-1}$ and if $\left(s_{0}, 0\right) \in U_{1}$ is a contracting attractor for all the trajectories in $t>0$, then it is an expanding attractor for those in $t<0$. For $t=0$ the behavior of the induced field is undetermined.

3. The k-dimensional case. This is treated in an analogous manner. Projective $k$-space $P^{\star}$ is given a differentiable structure by means of the neighborhoods $U_{i}=\left\{l(y) \in P^{k} \mid \forall y \in l(y), j\right.$ $\left.\neq i,\left[y_{j} / y_{i}\right]<\infty\right\}$ and the maps

$$
z=\varphi_{i}(l(y))=\left(y_{1}, \cdots, \hat{y}_{i}, \cdots, y_{k+1}\right) / y_{i},
$$

where the symbol ${ }^{\wedge}$ indicates that the $i$ th element is deleted. Projection of the paths into $P^{k}$ is achieved by means of the function given by

$$
f(x)=\left\{l(y) \cong P^{k} \mid y_{i} / y_{k+1}=x_{i}, \quad i=1, \cdots, k\right\} .
$$

It follows from the previous equations that $z=\left(x_{1}, \cdots \hat{x}_{i}, \cdots x_{k}, 1\right) / x_{i}$, and then

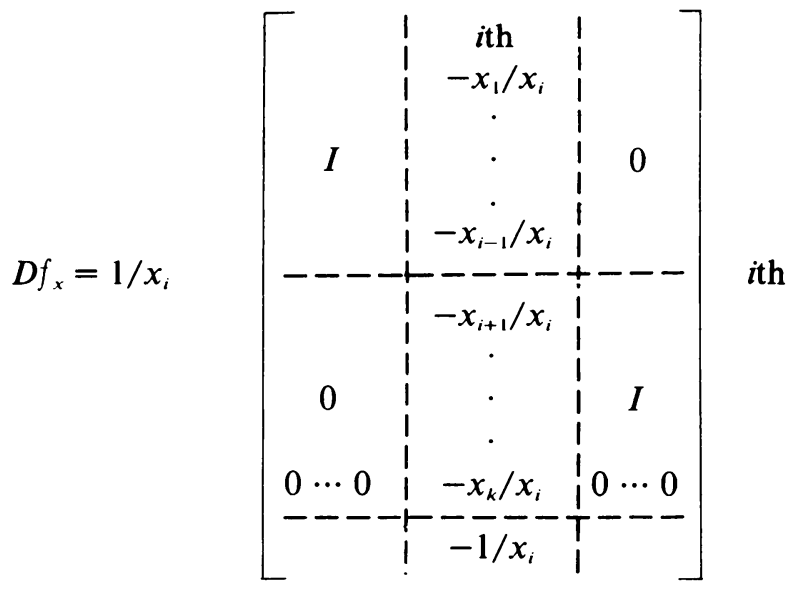

When $j$ and $l$ are such that $l \leq j<i \leq l<k$, and if $X=\left(P_{1}, \cdots, P_{k}\right)$, then we have

$$
\begin{aligned}
D f_{x}(X(x))= & \left(1 / x_{i}\right)^{2}\left(\cdots, x_{i} P_{(}(x)-x_{j} P_{i}(x), \cdots, x_{i} P_{l+1}(x)\right. \\
& \left.-x_{l+1} P_{i}(x), \cdots,-P_{i}(x)\right)
\end{aligned}
$$




$$
=\left(\frac{y_{k+1}}{y_{\mathrm{i}}}\right)^{2}\left[\cdots, \frac{y_{\mathrm{i}}}{y_{k+1}} P_{,}(\tilde{y})-\frac{y_{j}}{y_{k+1}} P_{i}(\tilde{y}), \cdots, \frac{y_{i}}{y_{k+1}} P_{l+1}(\tilde{y})-\frac{y_{l+1}}{y_{k+1}} P_{i}(\tilde{y}), \cdots,-P_{i}(\tilde{y})\right],
$$

where $\tilde{y}=\left(y_{1}, \cdots, y_{k}\right) / y_{k+1}$.

When $n$ is odd, application of the correcting factor $\left[y_{k+1}{ }^{2} /\left(y_{1}{ }^{2}+y_{2}{ }^{2}+\cdots+y_{k}{ }^{2+1}\right)\right]^{(n-1) / 2}$ $=\left[z_{k}{ }^{2} /\left(z_{1}{ }^{2}+\cdots+1+\cdots+z_{k}{ }^{2}\right)\right]^{(n-1 / 2}=z_{k}{ }^{n-1} / \Delta(z)_{n-1}$, where $\Delta(z)=\left(z_{1}{ }^{2}+\cdots+1+\cdots+\right.$ $\left.z_{k}^{2}\right)^{1 / 2}$, results in an induced field on $P^{k}$ given in $U_{i}$ by

$$
\frac{z_{k}{ }^{n}}{\Delta(z)^{n-1}}\left(\cdots, P_{j}(\tilde{z})-z_{j} P_{i}(\tilde{z}), \cdots, P_{l+1}(\tilde{z})-z_{l} P_{i}\left(\tilde{z}, \cdots,-z_{k} P_{i}(\tilde{z})\right),\right.
$$

where

$$
\tilde{z}=\left(z_{1}, \cdots, 1, \cdots, z_{k-1}\right) / z_{k} \text {. }
$$

As in the two-dimensional case, it is easy to see that the set $\left.\{l(y) \in P k\} y_{k+1}=0\right\}$ is invariant.

4. Example. We shall consider van der Pol's equation

$$
\ddot{x}+\epsilon\left(x^{2}-1\right) \dot{x}+x=0, \quad \epsilon>0
$$

or the equivalent vector field

$$
X(x, y)=\left(y, \epsilon\left(1-x^{2}\right) y-x\right)
$$

which is of degree $n=3$.

Except for the factor $1 / \Delta(s, t)^{2}$, which is always positive and does not change the trajectories of the field on $P^{2}$, Eqs. (6) and (7) yield

$$
t^{3}\left[\epsilon\left(1-\frac{1}{t^{2}}\right) \frac{s}{t}-\frac{1}{t}-s \frac{1}{t},-t \frac{s}{t}\right]=\left[\epsilon\left(t^{2}-1\right) s-t^{2}-s t^{2},-s t^{3}\right]
$$

and

$t^{3}\left[\frac{1}{t}-s \epsilon\left(1-\frac{s^{2}}{t^{2}}\right) \frac{1}{t}+s \frac{s}{t},-t \epsilon\left(1-\frac{s^{2}}{t^{2}}\right) \frac{1}{t}+\frac{s}{t}\right]=\left[t^{2}-\epsilon s\left(t^{2}-s^{2}\right)+s^{2} t^{2},-\epsilon t\left(t^{2}-s^{2}\right)+s t^{3}\right]$.

Both (16) and (17) have only one critical point, the origin. It is well known that $(0,0)$ is a saddle point of (16) and an expanding node of (17), and that the graphical representation of the induced field in a neighborhood of infinity is as in Fig. 1. $P^{2}$ is represented by the closed disk with opposite points on the circumference identified. See Gomory [4] for details.

Next, we shall focus our attention on the behavior of (17) in a neighborhood of $(0,0)$. We shall determine a subset of the region of attraction of this node as we move along trajectories in the direction of negative time. For this purpose we shall use the function $V$ : $R^{2} \rightarrow R$ given by

$$
V(s, t)=\left(s^{2}+t^{2}\right) / 2 .
$$

Along the trajectories of (17) we have

$$
\begin{aligned}
\dot{V}(s, t)=s \dot{s}+t \dot{t} & =s\left[t^{2}-\epsilon s\left(t^{2}-s^{2}\right)+s^{2} t^{2}\right]+t\left[-\epsilon t\left(t^{2}-s t^{3}\right)+s t^{3}\right] \\
& =s t^{2}+s^{3} t^{2}+s t^{4}+\epsilon s^{4}-\epsilon t^{4} .
\end{aligned}
$$




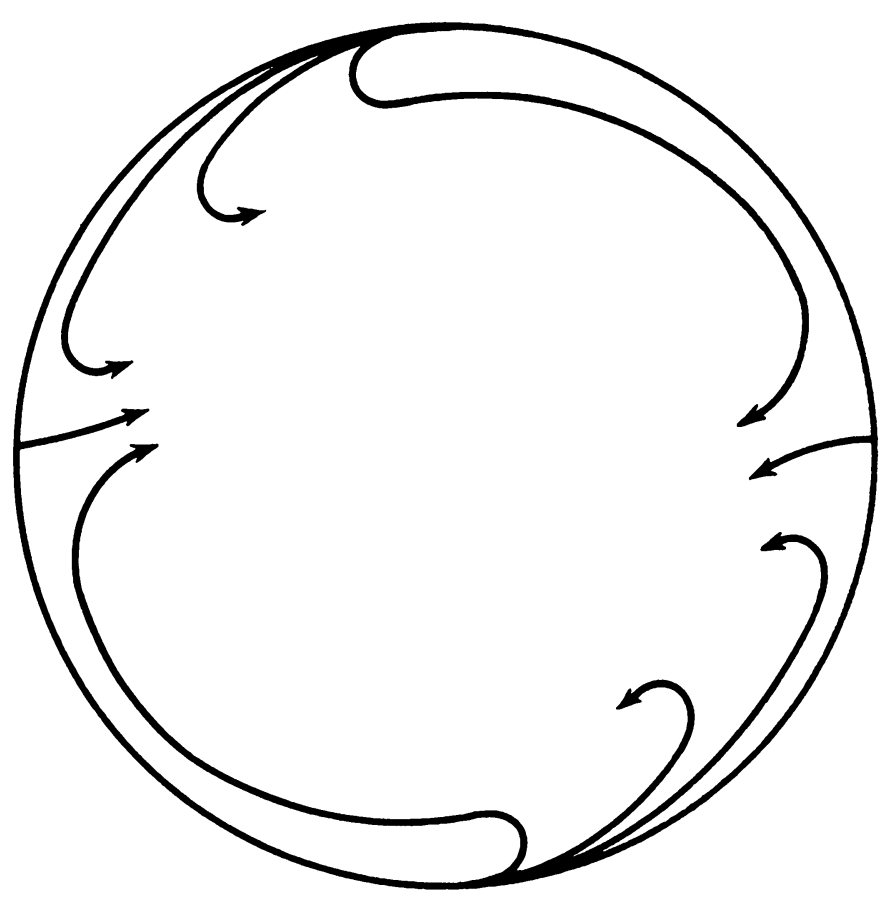

FIG. 1.

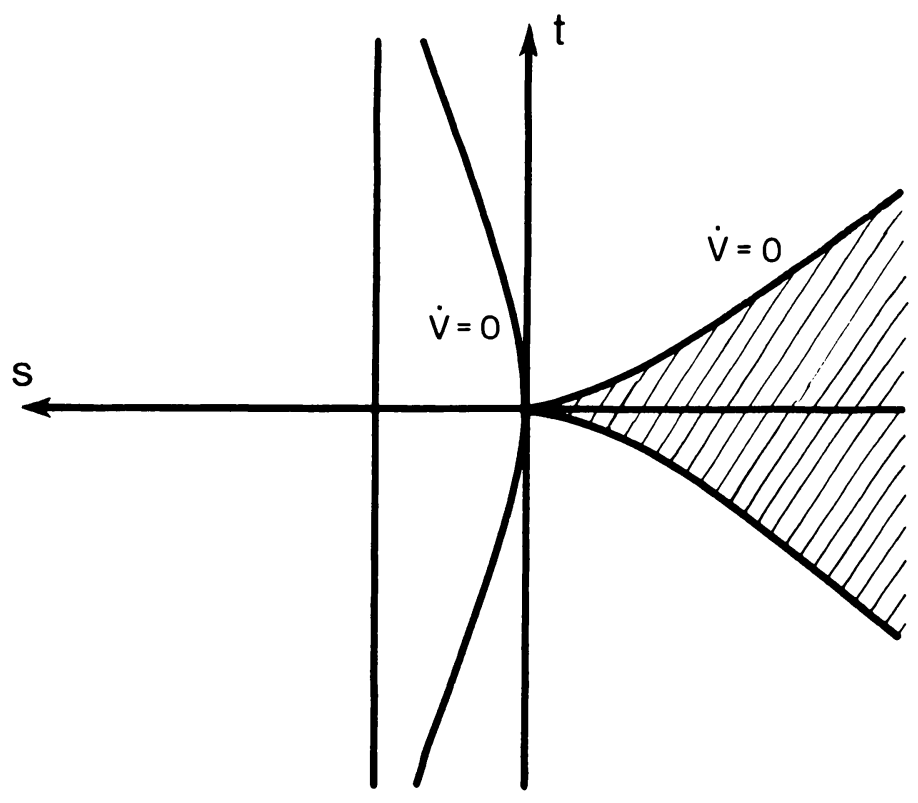

FIG. 2. 


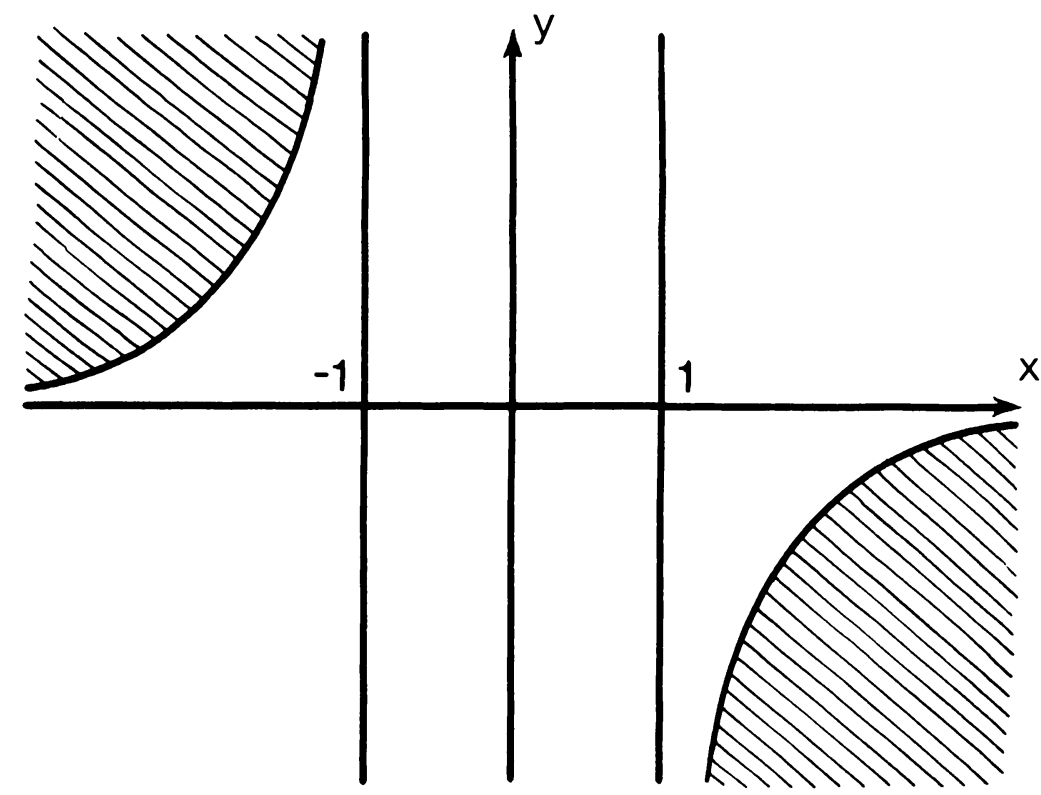

FIG. 3.

The curve $\dot{V}=0$ is represented in Fig. 2. Its only asymptote is $s=\epsilon$ and the tangents at the origin are $s=0$ and $t=0 . \dot{V}$ is positive in the shaded region. Notice that $\dot{V}=0$ is the locus of those points where the trajectories are tangent to the circles $s^{2}+t^{2}=r^{2}$. In fact, for $s<0$, the trajectories are transversal to the curve $\dot{V}=0$, entering the shaded ragion as we move against time. For $t>0$, this is so because the slope of any trajectory is positive at the point where it crosses $\dot{V}=0$, while on this curve implicit differentiation gives

$$
\frac{d t}{d s}=\frac{\left(1+3 s^{2}\right) t^{2}+t^{4}+4 \epsilon s^{3}}{4 t^{3}(\epsilon-s)-2 s t\left(1+s^{2}\right)}
$$

For $s<0, t\rangle 0$ the denominator is positive. The fact that the numerator is negative can be shown in a variety of ways. For example, when $s<0$ we have

$$
\begin{aligned}
& \dot{V}=0 \Rightarrow s t^{2}+s^{3} t^{2}+\epsilon s^{4}>0 \Rightarrow t^{2}<-\epsilon s^{3} /\left(1+s^{2}\right), \\
& \dot{V}=0 \Rightarrow s^{4}-t^{4}>0 \Rightarrow t^{2}<s^{2}, \\
& \dot{V}=0 \Rightarrow \epsilon s^{4}+s^{3} t^{2}>0 \Rightarrow t^{2}<-\epsilon s .
\end{aligned}
$$

The last two of these expressions imply that $t^{4}<-\epsilon s^{3}$. This and the first inequality above imply that

$$
\left(1+3 s^{2}\right) t^{2}+t^{4}+4 \epsilon s^{3}<-\epsilon s^{3}\left(1+3 s^{2}\right) /\left(1+s^{2}\right)-\epsilon s^{3}+4 \epsilon s^{3}=2 \epsilon s^{3} /\left(1+s^{2}\right)<0 .
$$

For $t<0$ an identical result is obtained by symmetry.

If follows that $V$ is a Liapunov function for the field

$$
-\left[t^{2}-\epsilon s\left(t^{2}-s^{2}\right)+s^{2} t^{2},-\epsilon t\left(t^{2}-s^{2}\right)+s t^{3}\right]
$$


on the set

$$
G=\left\{(s, t) \in R^{2} \mid s<0, \dot{V}(s, t)>0, s^{2}+t^{2}<r^{2}\right\},
$$

and that any trajectory starting in $G$ remains in $G$. Since $(0,0)$ is the only invariant set in $\{(s, t) \in \bar{G} \mid \dot{V}=0\}$, and since $r$ is arbitrary, it follows that $(0,0)$ is the $\omega$-limit set of any trajectory of this field starting in the set $S=\left\{(s, t) \in R^{2} \mid s<0, \dot{V}(s, t)>0\right\}$. That is, $(0,0)$ is the $\alpha$-limit set of any trajectory of (17) starting in $S$. As a consequence, the unique limit cycle of (15) cannot pass through $S$.

If we recall that $(s, t)=(x, 1) / y$ in $U_{2}$, the subset of the plane corresponding to $S$ is

$$
\left\{(x, y) \in R^{2} \mid x / y<0, x\left(1+x^{2}+y^{2}\right)+\epsilon\left(x^{4}-1\right)=0\right\} .
$$

It is represented by the shaded region in Fig. 3.

Also, recall that the origin of $R^{2}$ is the $\alpha$-limit set of all the trajectories of (15) starting inside the unit circle.

\section{REFERENCES}

[1] H. Poincaré, Mémoire sur les courbes définies par une equation différentielle, J. Mathématiques 7, 375-422 (1881)

[2] E. A. González-Velasco, Generic properties of polynomial vector fields at infinity, Trans. Amer. Math. Soc. 143, 201-222 (1969)

[3] S. Lefschetz, Differential equations: geometric theory, Interscience, New York, 1963.

[4] R. E. Gomory, Critical points at infinity and forced oscillation, in Contributions to the theory of nonlinear oscillations, Vol. III, S. Lefschetz, ed., Princeton University Press, Princeton, N. J., 1956 